\title{
Algorithmic Development of Power Series
}

\author{
Wolfram Koepf \\ Fachbereich Mathematik, Freie Universität Berlin \\ Arnimallee 3, W-1000 Berlin 33, Germany
}

\begin{abstract}
There is a one-to-one correspondence between formal power series (FPS) $\sum_{k=0}^{\infty} a_{k} x^{k}$ with positive radius of convergence and corresponding analytic functions. Since a goal of Computer Algebra is to work with formal objects and preserve such symbolic information, it should be possible to automate conversion between these forms in Computer Algebra Systems (CASs). However, only MACsYMA provides a rather limited procedure powerseries to calculate FPS from analytic expressions in certain special cases.

We present an algorithmic approach to compute an FPS, which has been implemented by the author and A. Rennoch in Mathematica, and by D. Gruntz in MAPLE. Moreover, the same algorithm can be reversed to calculate a function that corresponds to a given FPS, in those cases when an initial value problem for a certain ordinary differential equation can be solved.

Further topics of application like infinite summation, and asymptotic expansion are presented.
\end{abstract}

\section{Introduction}

We consider formal power series (FPS) of the form

$$
F:=\sum_{k=0}^{\infty} a_{k} x^{k}
$$

with coefficients $a_{k} \in \mathbb{C}\left(k \in \mathbb{N}_{0}\right)$. All the algebraic operations for FPS like addition, multiplication, division, and substitution, can be done by finite algorithms if one truncates the resulting FPS, i.e. only evaluates the first $N$ coefficients of it (where $N$ is an arbitrary fixed positive integer), which gives a truncated power series. These algorithms are implemented in certain Computer Algebra Systems (CAS), e.g. in Axiom ${ }^{1}$, Macsyma $^{2}$, Maple $^{3}$, Mathematica $^{4}$, and Reduce ${ }^{5}$ (see [1], [10], [11], [16], and [7], respectively).

\footnotetext{
1 AxIOM is a trademark of the Numerical Algorithms Group Ltd.

2 MACSYMA is a registered trademark of Symbolics, Inc.

${ }^{3}$ MAPle is a registered trademark of Waterloo Maple Software.

4 Mathematica is a registered trademark of Wolfram Research, Inc.

${ }^{5}$ REDUCE is a trademark of the RAND Corp.
} 
Moreover, all CAS provide a procedure to find a truncated power series expansion for a function $f$. By Taylor's Theorem the power series coefficients of a function $f$ can be calculated by the formula

$$
a_{k}:=\frac{f^{(k)}(0)}{k !},
$$

which provides an algorithmic procedure to calculate a truncated power series of certain degree $N$. The Taylor algorithm, however, does not generate an explicit formula for $a_{k}$. Moreover it generally has exponential complexity in the order $N$ : The differentiation of a product by the product rule may generate $2^{N}$ summands. Furthermore in the general case, e.g. for $f(x):=\frac{\sin x}{x}$, the evaluations $f^{(k)}(0)$ must be replaced by limits

$$
a_{k}:=\frac{\lim _{x \rightarrow 0} f^{(k)}(x)}{k !}
$$

whose evaluation are, in general, of exponential complexity as well. Moreover, the larger the number $N$, the larger the chance that the CAS even fails to evaluate these limits, when the expressions $f^{(k)}$ get more and more complicated.

For the following derivative free approach to generate the Taylor coefficients $a_{k}$ of a function $f$ recursively which is purely based on limits, similar restrictions apply. If

$$
f_{k}(x):=\left\{\begin{array}{cc}
f(x) & \text { if } k=0 \\
\frac{f_{k-1}(x)-\lim _{x \rightarrow 0} f_{k-1}(x)}{x} & \text { if } k \in \mathbb{N}
\end{array},\right.
$$

then the Taylor coefficients are given by

$$
a_{k}:=\lim _{x \rightarrow 0} f_{k}(x) .
$$

Most decisive, however, is the fact that none of these algorithms leads to a formula for $a_{k}$, i.e. the formal transformation "function expression of variable $x$ towards coefficient expression of variable $k$ " cannot be supported.

Thus in existing CAS the work with power series is restricted to truncated power series. Some of the systems, like Aхıом (previously SCR.ATCHPAD) and MAPLE, internally work with streams, and lazy evaluation, i.e. series objects are given by a finite number of initial terms, and an (internally used) formula to calculate further coefficients, see e.g. [12]. Infinite series representations, however, are not supported in these systems, either.

We shall give an outline of how to resolve these issues for FPS of some special types, including many special functions. Our procedure then produces the exact formal result, i.e. an explicit formula for the coefficients $a_{k}$. Obviously this solves the complexity problem, and as only a small number of limits have to be found, the chance to succeed is even larger than in the truncated case. 


\section{Laurent-Puiseux Series of Hypergeometric Type}

We require an assumption that every FPS $F$ has positive radius of convergence $r:=1 / \limsup _{k \rightarrow \infty}\left|a_{k}\right|^{1 / k}$. In this situation the FPS represents an analytic function $f(x)=\sum_{k=0}^{\infty} a_{k} x^{k}=: F$ in its disk of convergence $\mathbb{D}_{r}:=\{x \in \mathbb{C}|| x \mid<r\}$, i.e. its sum converges locally uniformly in $\mathbb{D}_{r}$ to $f$. So there is a one-to-one correspondence between the functions $f$ analytic at the origin and the FPS $F$ with positive radius of convergence represented by their coefficient sequences $\left(a_{k}\right)_{k \in \mathbb{N}_{0}}$. We denote this correspondence by $f \leftrightarrow F$. As we are interested in the conversion $f \leftrightarrow F$ the restriction to FPS with positive radius of convergence makes sense even though algebraically this restriction is not necessary.

To deal with many special functions, it is a good idea to consider the (generalized) hypergeometric series

$$
{ }_{p} F_{q}\left(\begin{array}{cccc}
a_{1} a_{2} & \cdots & a_{p} \\
b_{1} b_{2} & \cdots & b_{q}
\end{array} \mid x\right):=\sum_{k=0}^{\infty} \frac{\left(a_{1}\right)_{k} \cdot\left(a_{2}\right)_{k} \cdots\left(a_{p}\right)_{k}}{\left(b_{1}\right)_{k} \cdot\left(b_{2}\right)_{k} \cdots\left(b_{q}\right)_{k} k !} x^{k}
$$

where $(a)_{k}$ denotes the Pochhammer symbol (or shifled factorial) defined by

$$
(a)_{k}:=\left\{\begin{array}{cc}
1 & \text { if } k=0 \\
a \cdot(a+1) \cdots(a+k-1) & \text { if } k \in \mathbb{N}
\end{array}\right.
$$

Note that $\frac{(a)_{k}}{k !}=\left(\begin{array}{c}a+k-1 \\ k\end{array}\right)$, where $\left(\begin{array}{l}\alpha \\ k\end{array}\right)$ is the binomial coefficient

$$
\left(\begin{array}{l}
\alpha \\
k
\end{array}\right):=\left\{\begin{array}{cc}
1 & \text { if } k=0 \\
\frac{\alpha \cdot(\alpha-1) \cdots(\alpha-k+1)}{k !} & \text { if } k \in \mathbb{N}
\end{array},\right.
$$

and $k$ ! denotes the factorial

$$
k !:=\left\{\begin{array}{cc}
1 & \text { if } k=0 \\
1 \cdot 2 \cdots k & \text { if } k \in \mathbb{N}
\end{array} .\right.
$$

The coefficients $A_{k}$ of the hypergeometric series $\sum_{k=0}^{\infty} A_{k} x^{k}$ are the unique solution of the special recurrence equation (RE)

$$
A_{k+1}:=\frac{\left(k+a_{1}\right) \cdot\left(k+a_{2}\right) \cdots\left(k+a_{p}\right)}{\left(k+b_{1}\right) \cdot\left(k+b_{2}\right) \cdots\left(k+b_{q}\right)(k+1)} \cdot A_{k} \quad(k \in \mathbb{N})
$$

with the initial condition

$$
A_{0}:=1 \text {. }
$$

Note that $\frac{A_{k+1}}{A_{k}}$ is rational in $k$. Moreover if $\frac{A_{k+1}}{A_{k}}$ is a rational function $R(k)$ in the variable $k$ then the corresponding function $f$ is connected with a hypergeometric series; i.e., if $k=-1$ is a pole of $R$, then $f$ corresponds to a hypergeometric series evaluated at some point $A x$ (where $A$ is the quotient of the leading coefficients 
of the numerator and the denominator of $R$ ); whereas, if $k=-1$ is no pole of $R$, then $f$ may be furthermore shifted by some factor $x^{s}(s \in \mathbb{Z})$.

We further mention that the function $f$ corresponding to the hypergeometric series

$$
f \leftrightarrow F:={ }_{p} F_{q}\left(\begin{array}{llll}
a_{1} & a_{2} & \cdots & a_{p} \\
b_{1} & b_{2} & \cdots & b_{q}
\end{array} \mid x\right)
$$

satisfies the differential equation (DE)

$$
\theta\left(\theta+b_{1}-1\right) \cdots\left(\theta+b_{q}-1\right) f=x\left(\theta+a_{1}\right) \cdots\left(\theta+a_{p}\right) f
$$

where $\theta$ is the differential operator $x \frac{d}{d x}$. An inspection of the hypergeometric $\mathrm{DE}(2)$ shows that it is of the form $(Q:=\max \{p, q\}+1)$

$$
\sum_{j=0}^{Q} \sum_{l=0}^{Q} c_{l j} x^{l} f^{(j)}=0
$$

with certain constants $c_{l j} \in \mathbb{C}(l, j=0, \ldots, Q)$. Because of their importance in our development, we call a DE of the form (3), i.e. a homogeneous linear DE with polynomial coefficients, simple. We will show the existence of a simple DE for a more general family of functions.

It is remarkable that many elementary functions can be represented by hypergeometric series.

$$
\begin{aligned}
& (1+x)^{\alpha} \leftrightarrow{ }_{1} F_{0}(-\alpha \mid x), \quad e^{x} \leftrightarrow{ }_{0} F_{0}(x), \quad-\ln (1-x) \leftrightarrow x \cdot{ }_{2} F_{1}\left(\begin{array}{cc}
1 & 1 \\
2
\end{array} \mid x\right), \\
& \sin x \leftrightarrow x \cdot{ }_{0} F_{1}\left(3 / 2 \mid-\frac{x^{2}}{4}\right), \quad \cos x \leftrightarrow{ }_{0} F_{1}\left(1 / 2 \mid-\frac{x^{2}}{4}\right), \\
& \arcsin x \leftrightarrow x \cdot{ }_{2} F_{1}\left(\begin{array}{c}
1 / 21 / 2 \\
3 / 2
\end{array} \mid x^{2}\right), \quad \arctan x \leftrightarrow x \cdot{ }_{2} F_{1}\left(\begin{array}{c}
1 / 21 \\
3 / 2
\end{array} \mid-x^{2}\right) .
\end{aligned}
$$

Note that an FPS of the form $F\left(x^{m}\right)$ is called $m$-fold symmetric. Even functions are 2-fold symmetric and odd functions are shifted 2-fold symmetric functions.

By the above examples one is led to the following more general definition. First we extend the considerations to formal Laurent-Puiseux series (LPS) with a representation

$$
F:=\sum_{k=k_{0}}^{\infty} a_{k} x^{k / n} \quad\left(a_{k_{0}} \neq 0\right)
$$

for some $k_{0} \in \mathbb{Z}$, and $n \in \mathbb{N}$. LPS are formal Laurent series, evaluated at $\sqrt[n]{x}$. A formal Laurent series $(n=1)$ is a shifted FPS, and corresponds to a meromorphic $f$ with a pole of order $-k_{0}$ at the origin. The number $n$ in development (4) is called the Puiseux number of (the given representation of) $f$.

Definition (Functions of hypergeometric type). An LPS $F$ with representation (4) - as well as its corresponding function $f-$ is called to be of 
hypergeometric type if it has a positive radius of convergence, and if its coefficients $a_{k}$ satisfy a RE of the form

$$
\begin{aligned}
a_{k+m} & =R(k) a_{k} & & \text { for } k \geq k_{0} \\
a_{k} & =A_{k} & & \text { for } k=k_{0}, k_{0}+1, \ldots, k_{0}+m-1
\end{aligned}
$$

for some $m \in \mathbb{N}, A_{k} \in \mathbb{C}\left(k=k_{0}+1, k_{0}+2, \ldots, k_{0}+m-1\right), A_{k_{0}} \in \mathbb{C} \backslash\{0\}$, and some rational function $R$. The number $m$ is then called the symmetry number of (the given representation of) F. A RE of type (5) is also called to be of hypergeometric type. $\triangle$

We want to emphasize that the above terminology of functions of hypergeometric type is definitely more general than the terminology of a generalized hypergeometric function. It covers e.g. the function $\sin x$ which is not a generalized hypergeometric function as obviously no RE of the type (5) holds for its series coefficients with $m=1$. So $\sin x$ is not of hypergeometric type with symmetry number 1 ; it is, however, of hypergeometric type with symmetry number 2 . A more difficult example of the same kind is the function $e^{\arcsin x}$ which is neither even nor odd, and nevertheless turns out to be of hypergeometric type with symmetry number 2, too (see [8], Sect. 9). Further functions like $\frac{\sin x}{x^{5}}$ are covered by the given approach. Moreover the terminology covers composite functions like $\sin \sqrt{x}$, which do not have a Laurent, but a Puiseux series development.

If $F$ is $m$-fold symmetric, then there is a hypergeometric representation (5) with symmetry number $m$, whereas such a representation does not guarantee any symmetry. In fact, if $F$ is of hypergeometric type with symmetry number $j$, then it is of hypergeometric type with each multiple $m j(m \in \mathbb{N})$ of $m$ as symmetry number since by induction we get the RE

$$
a_{k+j m}=R(k) R(k+j) R(k+2 j) \cdots R(k+(m-1) j) a_{k},
$$

and $R(k) R(k+j) R(k+2 j) \cdots R(k+(m-1) j)$ is rational, too. In particular, each hypergeometric type function with symmetry number $j=1$ is of hypergeometric type for arbitrary symmetry number $m$.

On the other hand, it is clear that each LPS with symmetry number $m$, and Puiseux number $n$, can be represented as the sum of $n m$ shifted $m$-fold symmetric functions.

The derivative and the antiderivative of an LPS given by (4) are given by the rules

$$
\begin{gathered}
F^{\prime}:=\frac{1}{n} \sum_{k=k_{0}}^{\infty} k a_{k} x^{k / n-1}=\frac{1}{n} \sum_{k=k_{0}-1}^{\infty}(k+1) a_{k+1} x^{k / n}, \\
\int F:=\sum_{\substack{k=k_{0} \\
k \neq-1}}^{\infty} \frac{a_{k}}{k / n+1} x^{k / n+1}+a_{-1} \ln x=n \sum_{\substack{k=k_{0}+1 \\
k \neq 0}}^{\infty} \frac{a_{k-1}}{k} x^{k / n}+a_{-1} \ln x .
\end{gathered}
$$

Now we give a list of transformations on LPS that preserve the hypergeometric type. 
Lemma Let $F$ be an LPS of hypergeometric type with representation (4). Then
(a) $x^{j} F(j \in \mathbb{N})$,
(b) $F / x^{j} \quad(j \in \mathbb{N})$,
(c) $F(A x)(A \in \mathbb{C})$,
(d) $F\left(x^{\frac{p}{q}}\right)(p, q \in \mathbb{N})$,
(e) $\frac{F(x) \pm F(-x)}{2}$,
(f) $F^{\prime}$

are of hypergeometric type, too. If $a_{-1}=0$, then also

(g) $\int F$ is of hypergeometric type.

For a proof of this Lemma we refer to ([8], Lemma 2.1, Theorem 8.1). We note that as $\cos x=\frac{e^{i x}+e^{-i x}}{2}$ and $\sin x=\frac{e^{i x}-e^{-i x}}{2 i}$, a combination of (c) and (e) shows that the hypergeometric type of $\cos x$ and $\sin x$ follows from that of the exponential function.

We remark further that one can extend the definition of functions of hypergeometric type to include also the functions defined in (g) for arbitrary LPS (see [8], Sect. 8). Note that because of the logarithmic terms these functions, in general, do not represent LPS.

It is essential for our development that functions of hypergeometric type satisfy a simple DE.

Theorem Each LPS of hypergeometric type satisfies a simple DE.

Proof. Let $F$ be given by

$$
F:=\sum_{k=k_{0}}^{\infty} a_{k} x^{k / n} \quad\left(a_{k_{0}} \neq 0\right) .
$$

Define the differential operator $\theta_{n}:=n x \frac{d}{d x}$ working on a function of variable $x$. $\theta_{n}$ has the property

$$
\theta_{n} F=\sum_{k=k_{0}}^{\infty} k a_{k} x^{k / n},
$$

and by induction for all $j \in \mathbb{N}$

$$
\theta_{n}^{j} F=\sum_{k=k_{0}}^{\infty} k^{j} a_{k} x^{k / n} .
$$

This shows that moreover, if $P$ is any polynomial, we may formally write

$$
P(\theta) F=\sum_{k=k_{0}}^{\infty} P(k) a_{k} x^{k / n} .
$$

This commuting property is the reason why the differential operator $\theta_{n}$ is much more appropriate for the current discussion than the usual differential operator $\frac{d}{d x}$.

If the representation of $F$ has symmetry number $m$, we know that there is also a representation with symmetry number $n m$

$$
Q(k) a_{k+n m}=P(k) a_{k}
$$


with two polynomials $P$, and $Q$, and we may assume without loss of generality that the polynomials $P$ and $Q$ are chosen such that $Q\left(k_{0}-1\right)=Q\left(k_{0}-2\right)=\cdots=$ $Q\left(k_{0}-n m\right)=0$. This goal can be reached by multiplying both $P$ and $Q$ with the factors $\left(k-k_{0}+j\right)(j=1, \ldots, n m)$. From (6) and (7) we get

$$
\begin{aligned}
Q\left(\theta_{n}-n m\right) F & =\sum_{k=k_{0}}^{\infty} Q(k-n m) a_{k} x^{k / n} \quad \text { by }(6), \text { as } Q \text { is a polynomial } \\
& =\sum_{k=k_{0}+n m}^{\infty} Q(k-n m) a_{k} x^{k / n} \text { as } Q\left(k_{0}-1\right)=\cdots=Q\left(k_{0}-n m\right)=0 \\
& =\sum_{k=k_{0}}^{\infty} Q(k) a_{k+r m} x^{k / n+m} \quad \text { by an index shift } \\
& =x^{m} \sum_{k=k_{0}}^{\infty} P(k) a_{k} x^{k / n} \quad \text { by }(7) \\
& =x^{m} P(\theta) F \quad \text { by }(6) \text { again. }
\end{aligned}
$$

This represents a DE for $F$ which turns out to be of form (3). Note that for $m=1, n=1$, and $k_{0}=0$ we have exactly (2).

Now assume, a function $f$ representing an LPS is given. In order to find the coefficient formula, it is a reasonable approach to search for its DE, to transfer this DE into its equivalent RE, and you are done by an adaption of the coefficient formula for the hypergeometric function corresponding to the transformations given in the Lemma. It turns out that this procedure can be handled by an algebraic algorithm.

\section{The First Conversion Procedure}

There are two obvious transformation procedures: $f \mapsto F$ and $F \mapsto f$. At the moment we want to emphasize on the first situation. This transformation procedure $f \mapsto$ powerseries $(f, \mathbf{x}, \mathbf{x} 0)$ is implemented in MACsYMA (see [10]). The implementation in MACSYMA, however, uses heuristic maneuvers, and is not based on an algorithm. It fails to convert many important functions with a simple development like e.g.

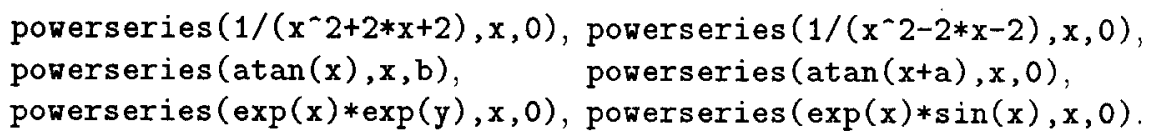

Here we present an algorithm corresponding to a function call of the form PowerSeries $[f, x, 0]$ - we use Mathematica syntax as our implementation is written in Mathematica language (see [16]) - i.e. producing a LaurentPuiseux series expansion of the function $f$ with respect to the variable $x$. 
Algorithm (for PowerSeries $[f, x, 0]$ )

(1) Rational functions (for details, see ([8], Sect. 4))

If $f$ is rational in $x$, then use the rational algorithm by calculating a complex partial fraction decomposition of $f$ (see e.g. [14], p. 171) which can be algorithmically done at least if the denominator has a rational factorization. Finally expand termwise by the binomial series.

(2) Find a simple DE (for details, see ([8], Sect. 5), where we prove that this procedure always succeeds in finding the simple $\mathrm{DE}$ of lowest degree for $f$ )

(a) Fix a number $N_{\max } \in \mathbb{N}$, the maximal order of the DE searched for; a suitable value is $N_{\max }:=4$.

(b) Set $N:=1$.

(c) Calculate $f^{(N)}$; either, if the derivative $f^{(N)}$ is rational, apply the rational algorithm, and integrate;

(d) or find a simple DE for $f$ of order $N$

$$
\sum_{j=0}^{N} p_{j} f^{(j)}=0
$$

where $p_{j}(j=0, \ldots, N)$ are polynomials in the variable $x$. Therefore decompose the expression

$$
f^{(N)}(x)+A_{N-1} f^{(N-1)}(x)+\cdots+A_{0} f(x)
$$

in elementary summands (with respect to the constants $A_{0}, \ldots, A_{N-1}$ ). Test, if the summands contain exactly $N$ rationally independent expressions. (Two expressions are called rationally independent if their ratio is not rational.) Just in that case there exists a solution as follows: Sort with respect to the rationally independent terms and create a system of linear equations by setting their coefficients to zero. Solve this system for the numbers $A_{0}, A_{1}, \ldots, A_{N-1}$. Those are rational functions in $x$, and there exists a unique solution. After multiplication by the common denominator of $A_{0}, A_{1}, \ldots, A_{N-1}$ you get the DE searched for.

(e) If (d) was not successful, then increase $N$ by one, and go back to (c), until $N=N_{\max }$.

(3) Find the corresponding RE (for details, see ([8], Sect. 6))

Suppose you found a simple DE in step (2), then transfer it into a RE for the coefficients $a_{k}$. The RE is then of the special type

$$
\sum_{j=0}^{M} P_{j} a_{k+j}=0,
$$

where $P_{j}(j=0, \ldots, M)$ are polynomials in $k$, and $M \in \mathbb{N}$. This is done by the substitution

$$
x^{l} f^{(j)} \mapsto(k+1-l)_{j} \cdot a_{k+j-l}
$$

into the DE. 
(4) Type of RE (for details, see ([8], Sect. 7))

Determine the type of the RE according to the following list

(a) If the RE (8) contains only two summands then $f$ is of hypergeometric type, and an explicit formula for the coefficients can be found by the hypergeometric coefficient formula (1), and some initial conditions.

(b) If the $\mathrm{DE}$ has constant coefficients $\left(c_{j} \in \mathbb{C}(j=0, \ldots, N)\right)$

$$
\sum_{j=0}^{N} c_{j} f^{(j)}=0,
$$

then $f$ is of exp-like type. In this case the substitution $b_{k}:=k ! \cdot a_{k}$ leads to the RE

$$
\sum_{j=0}^{N} c_{j} b_{k+j}=0,
$$

which has the same constant coefficients as the DE, and can be solved by a known algebraic scheme using the first $N$ initial coefficients.

(c) If the RE is none of the above types, try to solve it by other known RE solvers (a few of which are implemented in the Mathematica package DiscreteMath 'RSolve', e.g., and in MAPLE's rsolve).

The details of the single parts of the algorithm are presented in [8]. Here we prefer to give some examples for the use of the algorithm.

1. (Exp-like type case) Suppose $f(x)=e^{x} \sin x$, so $f^{\prime}=e^{x}(\sin x+\cos x)$ and $f^{\prime \prime}=2 e^{x} \cos x$. The first step of the algorithm does not apply. In the second step for $N:=1$ the expression $A_{0}=-(1+\cot x)$ is not rational in $x$. For $N:=2$ we get the expression

$$
f^{\prime \prime}+A_{1} f^{\prime}+A_{0} f=2 e^{x} \cos x+A_{1} e^{x}(\sin x+\cos x)+A_{0} e^{x} \sin x .
$$

Under the summands

$$
2 e^{x} \cos x, A_{1} e^{x} \sin x, A_{1} e^{x} \cos x, A_{0} e^{x} \sin x
$$

there are exactly the two rationally independent terms $e^{x} \cos x$ and $e^{x} \sin x$. We set the coefficient sums of these expressions to zero. The linear equations system

$$
2+A_{1}=0, \quad A_{1}+A_{0}=0
$$

has the solution $A_{1}=-2, A_{0}=2$, and leads so to the DE for $f$

$$
f^{\prime \prime}-2 f^{\prime}+2 f=0 \text {. }
$$

This DE has constant coefficients, so $f$ is of exp-like type. For $b_{k}:=k ! a_{k}$ we have the RE

$$
b_{k+2}-2 b_{k+1}+2 b_{k}=0 \text {. }
$$


The initial conditions are

$$
b_{0}=a_{0}=f(0)=0, \quad \text { and } \quad b_{1}=a_{1}=\frac{f^{\prime}(0)}{1 !}=1 .
$$

For a RE with constant coefficients the setup $b_{k}:=\lambda^{k}$ leads to a solution. Possible values for $\lambda$ are solutions of the equation $\lambda^{k+2}-2 \lambda^{k+1}+2 \lambda^{k}=0$, or solutions of the equivalent characteristic equation

$$
\lambda^{2}-2 \lambda+2=0,
$$

and so the values $\lambda_{1,2}:=1 \pm i$. Superposition (the RE is linear) leads to the general solution $b_{k}=A \lambda_{1}^{k}+B \lambda_{2}^{k}$ with constants $A, B \in \mathbb{C}$. The initial conditions lead then to the linear equations system for $A$ and $B$

$$
0=b_{0}=A+B, \quad 1=b_{1}=A(1+i)+B(1-i),
$$

whose solution is $A=\frac{1}{2 i}, B=-\frac{1}{2 i}$. So we have finally

$a_{k}=\frac{b_{k}}{k !}=\frac{1}{k !} \frac{(1+i)^{k}-(1-i)^{k}}{2 i}=\frac{1}{k !} \operatorname{Im}(1+i)^{k}=\frac{1}{k !} \operatorname{Im}\left(\sqrt{2} e^{i \frac{\pi}{4}}\right)^{k}=\frac{1}{k !} 2^{\frac{k}{2}} \sin \frac{k \pi}{4}$,

and

$$
F=\sum_{k=0}^{\infty} \frac{1}{k !} 2^{\frac{k}{2}} \sin \frac{k \pi}{4} x^{k} .
$$

2. (Hypergeometric type Puiseux series case) Suppose next $f(x)=$ $\sin \sqrt{x}$. Then $f^{\prime}=\frac{\cos \sqrt{x}}{2 \sqrt{x}}$, and $f^{\prime \prime}=-\frac{\sin \sqrt{x}}{4 x}-\frac{\cos \sqrt{x}}{4 \sqrt{x}^{3}}$. The second step of the algorithm leads to the DE for $f$

$$
4 x f^{\prime \prime}+2 f^{\prime}+f=0
$$

and the transformation via the rule (9) generates then the RE

$$
(2 k+1)(2 k+2) a_{k+1}+a_{k}=0,
$$

which is of hypergeometric type as only two summands occur. From the fact that the largest zero $k=-\frac{1}{2}$ of the polynomial in front of $a_{k+1}$ is nonintegral, we realize that we must consider a Puiseux series with Puiseux number 2 . So we make the transformation $b_{k}:=a_{k / 2}$, i.e. consider $g(x):=f\left(x^{2}\right)=$ $\sin x=\sum b_{k} x^{k}$ rather than $\sin \sqrt{x}=\sum b_{k} x^{k / 2}$, and get the hypergeometric type $\mathrm{RE}$

$$
(k+1)(k+2) b_{k+2}+b_{k}=0
$$

for $b_{k}$ with symmetry number $m=2$.

The fact that $g(0)=0$ tells us that $g$ is odd. We work with the FPS

$$
h(x):=\sum_{k=0}^{\infty} c_{k} x^{k}
$$


for which $g(x)=x h\left(x^{2}\right)$, and so $c_{k}=b_{2 k+1}$, leading to the RE for $c_{k}$

$$
c_{k+1}=-\frac{1}{4} \frac{1}{\left(k+\frac{3}{2}\right)(k+1)} c_{k} .
$$

The initial condition is $c_{0}=b_{1}=g^{\prime}(0)=1$, so that finally by (1)

$$
c_{k}=\frac{(-1)^{k}}{4^{k}\left(\frac{3}{2}\right)_{k} k !}=\frac{(-1)^{k}}{(2 k+1) !}
$$

and

$$
F=\sum_{k=0}^{\infty} \frac{(-1)^{k}}{(2 k+1) !} x^{k+1 / 2}
$$

3. (Hypergeometric type power series case) Now we consider the function

$$
f(x):=\int_{0}^{x} \frac{\operatorname{erf}(t)}{t} d t
$$

where erf denotes the error function

$$
\operatorname{erf}(x):=\frac{2}{\sqrt{\pi}} \int_{0}^{x} e^{-t^{2}} d t .
$$

Note that $f$ is not represented by means of elementary functions, however the algorithm still applies. We have $f^{\prime}=\frac{\operatorname{erf}(x)}{x}, f^{\prime \prime}=\frac{2}{\sqrt{\pi}} \frac{e^{-x^{2}}}{x}-\frac{\operatorname{erf}(x)}{x^{2}}$, and $f^{\prime \prime \prime}=-\frac{4}{\sqrt{\pi}} e^{-x^{2}}-\frac{4}{\sqrt{\pi}} \frac{e^{-x^{2}}}{x^{2}}+2 \frac{\operatorname{erf}(x)}{x^{3}}$, leading to the DE

$$
x f^{\prime \prime \prime}+\left(2 x^{2}+2\right) f^{\prime \prime}+2 x f^{\prime}=0,
$$

and thus the RE

$$
(k+2)^{2}(k+1) a_{k+2}+2 k^{2} a_{k}=0 .
$$

This is also of hypergeometric type, and the same argumentation as in Example 2 shows that $f$ is odd. We use the same substitutions and get for the coefficients $c_{k}$ of $h$

$$
c_{k+1}=-\frac{\left(k+\frac{1}{2}\right)^{2}}{\left(k+\frac{3}{2}\right)^{2}(k+1)} c_{k}
$$

with initial condition $c_{0}=a_{1}=f^{\prime}(0)=\frac{2}{\sqrt{\pi}}$, so that finally

$$
c_{k}=\frac{2}{\sqrt{\pi}} \frac{(-1)^{k}\left(\left(\frac{1}{2}\right)_{k}\right)^{2}}{\left(\left(\frac{3}{2}\right)_{k}\right)^{2} k !}=\frac{2}{\sqrt{\pi}} \frac{(-1)^{k}}{(2 k+1)^{2} k !}
$$

and

$$
F=\sum_{k=0}^{\infty} \frac{2}{\sqrt{\pi}} \frac{(-1)^{k}}{(2 k+1)^{2} k !} x^{2 k+1} .
$$


4. (Rational type case) Let's now look at $f(x)=\arctan x$. Here $f^{\prime}=\frac{1}{1+x^{2}}$ is rational. So we apply the rational algorithm (see ([8], Sect. 4)), and integrate. We find the complex partial fraction decomposition

$$
f^{\prime}(x)=\frac{1}{1+x^{2}}=\frac{1}{2}\left(\frac{1}{1+i x}+\frac{1}{1-i x}\right),
$$

from which we deduce for the coefficients $b_{k}$ of the derivative $F^{\prime}(x)=$ $\sum_{k=0}^{\infty} b_{k} x^{k}$ by using the binomial series that

$$
b_{k}=\frac{1}{2}\left(i^{k}+(-i)^{k}\right)=\frac{i^{k}}{2}\left(1+(-1)^{k}\right) .
$$

By the calculation

$$
b_{2 k+1}=\frac{i^{2 k+1}}{2}\left(1+(-1)^{2 k+1}\right)=0
$$

it follows that $F^{\prime}$ turns out to be even. Moreover

$$
b_{2 k}=\frac{i^{2 k}}{2}\left(1+(-1)^{2 k}\right)=(-1)^{k},
$$

so that by integration

$$
F=\sum_{k=0}^{\infty} \frac{(-1)^{k}}{2 k+1} x^{2 k+1} .
$$

We remark that the arctan function can also be handled by the hypergeometric procedure similarly as Example 2.

5. (Hypergeometric type Laurent series case) We consider $f(x):=$ $\frac{\arcsin ^{2} x}{x^{4}}$. Here the algorithm produces the DE

$$
\left(x^{5}-x^{3}\right) f^{\prime \prime \prime}+\left(15 x^{4}-12 x^{2}\right) f^{\prime \prime}+\left(61 x^{3}-36 x\right) f^{\prime}+\left(64 x^{2}-24\right) f=0,
$$

converting to the hypergeometric type RE

$$
(k+6)(k+5) a_{k+2}-(k+4)^{2} a_{k}=0
$$

with symmetry number 2 . It follows that $a_{-6}=a_{-5}=0$, and thus for all $k \leq-5$ we have $a_{k}=0$. Therefore we consider the shifted function $g(x):=x^{4} f(x)$ with the coefficients $b_{k}=a_{k-4}$ for which the RE

$$
b_{k+2}=\frac{k^{2}}{(k+2)(k+1)} b_{k}
$$

holds. The hypergeometric coefficient formula finally leads to

$$
F=\sum_{k=0}^{\infty} \frac{(k !)^{2} 4^{k}}{(2 k+1) !(k+1)} x^{2 k-2} .
$$




\section{Scope of the Algorithm}

In CAS's problems can be solved by means of

- implemented data bases, or

- algorithmic calculations.

In the first case, the implemented data base is fixed for all times until the software is released, whereas in the second case the use of the CAS generates a data base. Having an implementation of an algebraic algorithm makes a CAS an expert system: An arbitrary user may produce results, which had been unknown before, and can be added to a data base. Concerning the conversion of functions and power series [6] probably is the most exhaustive existing data base. It is a collection of numerical series, power series, products, and other material. It is easy to observe that most of its power series entries are of hypergeometric type, and so it is not surprising how many of them can be treated by our algorithm. The algorithm covers results about integrals that cannot be represented by elementary functions like Fresnel integrals, Bessel functions, and many other functions. More examples are given in ([8], Sect. 9), and will be published elsewhere [9].

When using [6] to find a power series representation, the scope is restricted to its finite contents, and moreover the success depends on the user's ability to find the entry he's searching for: The problem is thus converted into a search problem. On the other hand, the use of the CAS implementation of our algorithm does not have this kind of limitations. It is only a question of time when new results are discovered by its use.

\section{The Algorithm as a Simplifier}

One of the main questions of Computer Algebra is to decide whether a given expression algebraically is equivalent to zero or not. A simple example of this kind is the rational expression

$$
\frac{2}{1-x^{2}}-\frac{1}{1-x}-\frac{1}{1+x}
$$

which after an expansion with common denominator algebraically simplifies to zero. Much more difficult are nonrational algebraic or transcendental expressions like

$$
\begin{gathered}
\arcsin \frac{1}{3}+2 \arctan \frac{\sqrt{2}-\frac{\pi}{2}}{2}, \sqrt{x+1}+1-\sqrt{2 \sqrt{x+1}+x+2}, \\
\sqrt{\frac{1-\sqrt{1-x}}{x}}-\frac{\sqrt{1+\sqrt{x}}-\sqrt{1-\sqrt{x}}}{\sqrt{2 x}}, \\
\cos (4 \arccos x)-\left(1-8 x^{2}+8 x^{4}\right), \quad \text { or } \quad \cos (\arcsin x)-\sqrt{1-x^{2}},
\end{gathered}
$$

all of which turn out to equal zero. In general, it cannot be decided if a given transcendental expression is equivalent to zero. Our algorithm, however, may 
assist with this decision. Assume, an expression involving a variable $x$ is given, and we apply our algorithm to it. In principle, all expressions which are equivalent to zero, are of hypergeometric type; indeed, every polynomial, especially the zero polynomial, is of hypergeometric type. It may happen, however, that we cannot decide this since the hypergeometric type DE may not be found because of the lack of algebraic simplifications. If we are lucky, however, the expression is identified to be of hypergeometric type, in which case its series coefficients can be calculated, and are quotients of Pochhammer symbols. For this kind of expressions, however, it can be decided whether or not they are equivalent to zero, and so we will get the desired result. As examples, all above mentioned expressions that depend on $x$ are recognized by the algorithm to equal zero.

More results in this direction can be found in ([8], Sect. 10). We are convinced that the use of the algorithm will lead to new identities.

\section{The Second Conversion Procedure}

The algorithm presented has a natural inverse $F \mapsto f$, calculating the function $f$ from its LPS $F$. We omit the details of that procedure Convert $[F, \mathbf{x}]$ that converts an LPS $F$ into its equivalent function $f$ with respect to the variable $x$. Given a formula for the general coefficient $a_{k}$, the first step consists of finding a RE of the type (8). This step is algebraically equivalent to the search for the DE, presented in ([8], Sect. 5).

The next step is then the back-substitution that produces the left-hand side of the DE from the left-hand side of the RE, see ([8], Sect. 11).

The main part of the procedure Convert is to solve the finally generated simple DE together with some initial conditions. At the moment, with MATHEMATICA Version 2.0, this is, at least in the case of DE's of order greater than 2, in general beyond its capabilities. On the other hand, all but very few examples that we tested were solved by MACSYMA's ode procedure (version 417). By a theorem of Singer ([13], see e.g. [2], p. 192) there is an algorithm to decide whether the corresponding function has a representation in terms of elementary functions, in which case this representation is produced. It turns out, however, that the initial value problem may involve rather complicated nonlinear equations for the occuring integration constants.

This procedure Convert moreover is able to produce a closed form representation for (convergent) infinite sums

$$
\sum_{k=0}^{\infty} a_{k}
$$

whenever the numbers $a_{k}$ satisfy a homogeneous, linear RE with polynomial coefficients $P_{j}(j=0, \ldots, M)$

$$
\sum_{j=0}^{M} P_{j}(k) a_{k+j}=0
$$


and the generating function

$$
f(x):=\sum_{k=0}^{\infty} a_{k} x^{k}
$$

of the sequence $\left(a_{k}\right)$ has a representation by means of elementary functions. Convert produces $f$, and finally

$$
\sum_{k=0}^{\infty} a_{k}=\lim _{x \uparrow 1} f(x)
$$

by Abel's continuity theorem for power series (see e.g. [14], p. 149).

This algorithm should be compared with the Gosper algorithm ([3], see e.g. [5], § 5.7) which finds a closed form representation for a sum $A_{n}=\sum_{k=0}^{n} a_{k}$ with variable upper bound, in the special case that $A_{n}$ is the $n^{\text {th }}$ term of a hypergeometric function. In this case the generating function of the sequence $\left(a_{k}\right)$ turns out to be hypergeometric, too.

Our procedure does not generate closed forms for sums with variable upper bound, except in the special case that the given sequence $\left(a_{k}\right)$ satisfies $a_{k}=0$ for $k>n$. This case, however, occurs e.g. when considering sums of products of binomial coefficients. Moreover, for infinite sums our algorithm has a much wider range of applicability.

We consider two examples.

1. (Indefinite summation) We search for a closed formula for $(n \in \mathbb{N})$

$$
A_{n}:=\sum_{k=0}^{n}\left(\begin{array}{l}
n \\
k
\end{array}\right) \text {. }
$$

As $n \in \mathbb{N}$, the coefficients $a_{k}:=\left(\begin{array}{l}n \\ k\end{array}\right)=0$ for $k>n$ so that we can consider $A_{n}$ as an infinite sum

$$
A_{n}=\sum_{k=0}^{\infty}\left(\begin{array}{l}
n \\
k
\end{array}\right),
$$

and the method applies. The first part of the procedure Convert produces the RE

$$
(k+1) a_{k+1}-(n-k) a_{k}=0
$$

for the coefficients of the generating function

$$
f(x):=\sum_{k=0}^{\infty}\left(\begin{array}{l}
n \\
k
\end{array}\right) x^{k}
$$

which is transferred by the back-substitution into the DE

$$
(1+x) f^{\prime}-n f=0
$$


for $f$. The initial value problem

$$
(1+x) f^{\prime}-n f=0, \quad f(0)=a_{0}=1
$$

has the solution

$$
f(x)=(1+x)^{n}
$$

and so we have finally

$$
A_{n}=\lim _{x \uparrow 1} f(x)=(1+1)^{n}=2^{n},
$$

the desired result.

2. (Infinite summation) As another example we consider the infinite sum which cannot be treated by Gosper's algorithm

$$
\sum_{k=0}^{\infty} a_{k}=\sum_{k=0}^{\infty} \frac{(-1)^{k}}{2 k+1}
$$

with generating function

$$
f(x):=\sum_{k=0}^{\infty} a_{k} x^{k}=\sum_{k=0}^{\infty} \frac{(-1)^{k}}{2 k+1} x^{k} .
$$

An application of the first part of procedure Convert produces the RE for the coefficients $a_{k}$

$$
(2 k+3) a_{k+1}+(2 k+1) a_{k}=0
$$

which holds for $k \geq 0$. If we multiply this by the factor $(k+1)$, the resulting RE

$$
(k+1)(2 k+3) a_{k+1}+(k+1)(2 k+1) a_{k}=0
$$

holds for all $k \in \mathbb{Z}$. The back-substitution yields the initial value problem

$$
\left(2 x+2 x^{2}\right) f^{\prime \prime}+(3+5 x) f^{\prime}+f=0, \quad f(0)=a_{0}=1, \quad f^{\prime}(0)=a_{1}=-\frac{1}{3},
$$

for $f$, which has the solution

$$
f(x)=\frac{\arctan \sqrt{x}}{\sqrt{x}} .
$$

Thus the original sum has the value

$$
\sum_{k=0}^{\infty} \frac{(-1)^{k}}{2 k+1}=\lim _{x \uparrow 1} f(x)=\arctan 1=\frac{\pi}{4} .
$$

We mention that the ability to deal with the conversion of series and generating functions algorithmically leads to the ability to produce binomial identities (see e.g. [15], $\S 4.3$ ), to solve recurrence equations (see e.g. [5], $\S 7.3$ ), and to solve problems in probability theory (see e.g. [5], § 8.3). 


\section{Asymptotic Series}

One more field where the method described can be used, are asymptotics (see e.g. [5], Chap. 9). Assume, a function $f$ on the real axis is given, and we are interested in an asymptotic expansion, i.e. a function $g$ for which

$$
\lim _{x \rightarrow \infty} \frac{f(\dot{x})}{g(x)}=1
$$

Asymptotic expansions by no means are unique; indeed each function is its own asymptotic expansion, but there may exist much simpler asymptotic expansions as well. It is a special property of a function $f$ if one of its asymptotic expansions forms a Laurent-Puiseux series

$$
F:=\sum_{k=k_{0}}^{\infty} a_{k}\left(\frac{1}{x}\right)^{k / n} \quad\left(a_{k_{0}} \neq 0\right),
$$

and if $f$ has such a Laurent-Puiseux asymptotic expansion (LPA), then it is unique. Note that as we consider only positive values $x$ (or complex values $x$ that lie in a certain sector) the LPA is only one-sided.

It is now easy to see that a slight modification of the algorithm PowerSeries can be used to produce the LPA of a function $f$ by the following procedure:

1. Consider $h(x):=f\left(\frac{1}{x}\right)$.

2. Find a DE for $h$, and a RE for its one-sided series coefficients

$$
h(x)=\sum_{k=k_{0}}^{\infty} a_{k} x^{k / n} \quad(x \geq 0)
$$

by the PowerSeries algorithm.

3. Find the corresponding initial values by taking one-sided limits, e.g.

$$
a_{0}:=\lim _{x \downarrow 0} h(x)
$$

and solve the RE.

4. Finally you have

$$
F=\sum_{k=k_{0}}^{\infty} a_{k}\left(\frac{1}{x}\right)^{k / n}
$$

We mention that the one-sided limits considered are complex limits if and only if the function $h(x):=x^{k_{0}} f\left(x^{n}\right)$ is analytic at $\infty$, and so the corresponding LPA of $h$ equals its power series representation there. Moreover the radius of convergence equals zero, and so $F$ is a divergent series, if one of the one-sided limits considered is not a complex limit.

Since Mathematica only supports the calculation of complex limits, there is no direct way for an implementation of this procedure. On the other hand, MAPLE has some capabilities to calculate one-sided limits, and thus the MAPLE implementation covers asymptotic series expansions.

We give some examples of this procedure. 
1. Suppose $f(x)=e^{x}(1-\operatorname{erf} \sqrt{x})$. The algorithm leads to the DE

$$
2 x^{3} h^{\prime \prime}+\left(2 x+3 x^{2}\right) h^{\prime}-h=0
$$

for $h(x)=f\left(\frac{1}{x}\right)=e^{1 / x}\left(1-\operatorname{erf} \frac{1}{\sqrt{x}}\right)$, and the transformation via the rule (9) generates then the RE

$$
a_{k+1}+k a_{k}=0,
$$

which is of hypergeometric type. The initial values lead then to the asymptotic series representation

$$
F=\frac{1}{\sqrt{\pi}} \sum_{k=0}^{\infty} \frac{(-1)^{k}(2 k) !}{4^{k} k !}\left(\frac{1}{x}\right)^{k+1 / 2}
$$

2. If $f(x):=x e^{-x} \operatorname{Ei}(x)$ where Ei denotes the exponential integral function

$$
\operatorname{Ei}(x):=\int_{-\infty}^{x} \frac{e^{t}}{t}
$$

with a Cauchy principal value taken. We get the DE

$$
x^{2} h^{\prime \prime}+(3 x-1) h^{\prime}+h=0
$$

for $h(x)=f\left(\frac{1}{x}\right)$, and the $\mathrm{RE}$

$$
a_{k+1}=(k+1) a_{k}
$$

for its coefficients, leading to the asymptotic expansion

$$
F=\sum_{k=0}^{\infty} k !\left(\frac{1}{x}\right)^{k}
$$

\section{References}

1. Axıом: User Guide. The Numerical Algorithms Group Ltd., 1991.

2. Davenport, J.H., Siret, Y., Tournier, E.: Computer-Algebra: Systems and algorithms for algebraic computation. Academic Press, 1988.

3. Gosper Jr., R.W.: Decision procedure for indefinite hypergeometric summation. Proc. Natl. Acad. Sci. USA 75 (1978) $40-42$.

4. Gradshteyn, I.S., Ryzhik, I.M.: Table of integrals, series, and products - corrected and enlarged version. Academic Press, New York-London, 1980.

5. Graham, R.L., Knuth, D.E., Patashnik, O.: Concrete Mathematics: A Foundation for Computer Science. Addison-Wesley Publ. Co., Reading, Massachusetts, 1988.

6. Hansen, E.R.: A table of series and products. Prentice-Hall, Englewood Cliffs, NJ, 1975.

7. Hearn, A.: Reduce User's manual, Version 3.4. The RAND Corp., Santa Monica, CA, 1987. 
8. Koepf, W.: Power series in Computer Algebra. J. Symb. Comp. 13 (1992) 581-603.

9. Koepf, W.: Examples for the algorithmic calculation of formal Puiseux, Laurent and power series (to appear).

10. MacSYMA: Reference Manual, Version 13. Symbolics, USA, 1988.

11. MAPLE: Reference Manual, fifth edition. Watcom publications, Waterloo, 1988.

12. Norman, A. C.: Computing with formal power series. Transactions on mathematical software 1, ACM Press, New York (1975) 346-356.

13. Singer, M. F.: Liouvillian solutions of $\boldsymbol{n}$-th order homogeneous linear differential equations. Amer. J. Math. 103 (1981) 661-682.

14. Walter, W.: Analysis I. Springer Grundwissen Mathematik 3, Springer-Verlag, Berlin-Heidelberg-New York-Tokyo, 1985.

15. Wilf, H.S.: Generatingfunctionology. Academic Press, Boston, 1990.

16. Wolfram, St.: MathematicA. A system for doing mathematics by Computer. Addison-Wesley Publ. Comp., Redwood City, CA, 1991. 